Pediatrics

\title{
Comparison between ActiGraph GT3X and ActivPAL to assess sedentary behavior during the school period
}

\author{
Luciana Leite Silva Barboza $^{1}$ (D), André Oliveira Werneck ${ }^{2}$ (D), David Ohara ${ }^{3}$ (D), \\ Enio Ricardo Vaz Ronque ${ }^{4}$ (D), Marcelo Romanzini ${ }^{4}$ (D), Danilo Rodrigues Pereira da Silva ${ }^{1,5}$ \\ ${ }^{1}$ Universidade Federal de Sergipe, Programa de Pós-Graduação em Educação Física, São \\ Cristóvão, SE, Brazil; ${ }^{2}$ Universidade de São Paulo, Faculdade de Saúde Pública, Departamento \\ de Nutrição, Núcleo de Pesquisas Epidemiológicas em Nutrição e Saúde, São Paulo, SP, Brazil; \\ ${ }^{3}$ Universidade Estadual de Santa Cruz, Departamento de Ciências da Saúde, Ilhéus, BA, Brazil; \\ ${ }^{4}$ Universidade Estadual de Londrina, Departamento de Educação Física, Centro de Educação \\ Física e Esporte, Londrina, PR, Brazil; ${ }^{5}$ Universidade Federal de Sergipe, Departamento de \\ Educação Física, São Cristóvão, SE, Brazil.
}

Associate Editor: Romulo A Fernandes (D, Universidade Estadual Paulista "Júlio de Mesquita Filho”, Presidente Prudente, SP, Brazil. E-mail: romulo_ef@yahoo.com.br.

\begin{abstract}
Aims: This study aimed to compare the sedentary time measured using the ActiGraph GT3X accelerometer with the measurement of sitting and standing time obtained by ActivPAL inclinometers. Methods: This was a cross-sectional study conducted with a sample of 60 schoolchildren (34 males) of one elementary public school in Brazil. The students used both an ActiGraph GT3X accelerometer and an ActivPAL inclinometer, concurrently, positioned at the beginning and removed at the end of the school shift, for four days. For analysis, paired Student's t-tests, Pearson's correlation coefficients, intraclass correlation coefficients, and Bland-Altman plots were used. Results: When comparing sedentary time with sitting time, although correlated $(\mathrm{r}=0.53 ; \mathrm{p}<0.001)$, the mean minutes were different (134.2 min/day in ActiGraph GT3X vs $120.3 \mathrm{~min} /$ day in ActivPAL; $\mathrm{p}<0.001)$, with a bias of $13.9 \mathrm{~min} /$ day. When comparing the measurement of sedentary time with the sum of the sitting time plus standing time, different mean minutes were also observed ( $134.2 \mathrm{~min} /$ day in ActiGraph GT3X vs $177.0 \mathrm{~min} /$ day in ActivPAL; $\mathrm{p}<0.001)$, and although the correlation was stronger $(\mathrm{r}=0.75 ; \mathrm{p}<0.001)$, the bias was higher $(-42.8 \mathrm{~min} /$ day $)$. Conclusion: Sedentary time derived from the ActiGraph GT3X device should be used with caution to evaluate sedentary behavior in a school setting and may be interpreted only as non-moving activities (stationary behavior).
\end{abstract}

Keywords: sedentary lifestyle, process measures, methods, instrumentation, child.

\section{Introduction}

Sedentary behavior (SB) is conceptualized as any waking behavior with an energy expenditure of up to 1.5 METs, adopting sitting, reclining, or lying positions ${ }^{1}$. Despite the well-known risk for chronic diseases derived from physical inactivity ${ }^{2}$, SB has been studied as an independent risk factor for the development of chronic non-communicable diseases and mortality ${ }^{3,4}$. In children and adolescents, the high prevalence of SB, especially on screens (television, computer, and video games), is associated with unfavorable body composition, higher clustered cardiometabolic risk scores, unfavorable behavioral conduct/pro-social behavior, lower fitness, low selfesteem $^{5}$, poor health-related quality of life $^{6}$, an increase in psychological illnesses such as depression, and decreased psychological well-being, such as life satisfaction and well-being ${ }^{7}$.
To objectively evaluate SB, portable devices such as inclinometers and accelerometers are widely used in scientific research ${ }^{8-10}$. One of the most used inclinometers is the ActivPAL, which consists of a small portable device that is fixed to the middle anterior region of the individual's thigh to record time spent in sitting/reclining, standing stationary, and walking activities ${ }^{11}$. Regarding accelerometers, the most popular are the ActiGraph GT3X and GT3X+ models, which are typically used at the height of the iliac crest and record information based on threeaxis acceleration, classifying activities as sedentary, light, moderate, or vigorous according to the pattern of acceleration $^{12}$.

Although hip-worn ActiGraph GT3X and GT3X+ accelerometer models do not differentiate body positions, these devices are widely used in studies to evaluate SB from counts, using specific cut-off points ${ }^{10,12}$. However, to differentiate SB from stationary activities, it is necessary 
to identify whether the individual is in a sitting/reclining or standing position ${ }^{13}$.

The measure of SB using accelerometers in children is particularly specific due to the distinct pattern of accelerations, in which younger children present more physical activity, with shorter bouts ${ }^{14}$, which consequently leads to a higher frequency of sedentary time breaks. Besides, when considering sedentary time, the use of acceleration as the only criterion could lead to a potential bias and consequently, overestimate SB, keeping in mind the difficulty of measuring standing time ${ }^{15,16}$. This is especially relevant given that the school period is composed of several activities practiced in a standing position inside classes, but without clear accelerations. Therefore, the use of a device that only takes into consideration the criterion acceleration could overestimate sedentary behavior even more than outside school ${ }^{17}$. Previous studies found that ActivPAL inclinometers were better for estimating standing time and sitting time than accelerometers (adopting direct observation as the gold standard method) ${ }^{18}$, as well as that sitting time estimates measured through inclinometers moderately agree with sitting time measures through accelerometers $^{16}$.

Considering the limitations of accelerometers to measure standing time based on acceleration, the sum of standing and sitting/reclining positions using inclinometers (stationary behavior) could be more associated with accelerometer-based SB than only the time in sitting/reclining positions. However, the association between stationary behavior and SB from accelerometers is still lacking. Moreover, the associations between the ActivPAL inclinometer and the ActiGraph GT3X accelerometer still warrant investigation considering school time in children at the beginning of elementary school. Therefore, we aimed to compare the sedentary time measured using the ActiGraph GT3X accelerometer with the measurement of sitting and standing time obtained by ActivPAL inclinometers.

\section{Methods}

\section{Study outline}

The present cross-sectional study used data from the baseline of the ERGUER/Aracaju Project, performed between April and June 2018, which is a controlled trial aimed at evaluating the effects of reducing sitting time at school on cognition and academic performance of students. The study was approved by the local ethics committee of the Federal University of Sergipe (Process Number: 2.587.676), and children received permission to participate from parents or guardians, through signing an informed consent form.

\section{Participants}

The sample consisted of schoolchildren from four classes of the 2nd year of one elementary public school in Aracaju/SE, a city located in the northeast of Brazil. The school and classes were chosen for convenience. Initially, all children from the four classes were invited to participate in the study $(n=100)$; however, only 61 children were authorized by their parents by signing the consent form. Of these, one child did not have data for at least four days of evaluation and the final sample consisted of 60 schoolchildren (34 males; mean age of $7.8 \pm 0.6$ years).

\section{Instrumentation and procedure}

The students used, concurrently, an accelerometer ActiGraph GT3X and an inclinometer ActivPAL, placed in position by the research team (professionals and students of Physical Education, appropriately trained) at the beginning of the school shift $(7 \mathrm{am})$ and removed at the end of the school shift (11 am), for four days, considering the means of each child for each device. The days of use of the devices were preferably consecutive, except when the children were absent and used the devices on another nonconsecutive day, to complement the four days of use for all children. The time of use of the two devices was equated using as a reference the start time of recording of the inclinometers and the end time of recording of the accelerometers, for each day of recording.

The accelerometer (GT3X - ActiGraph Inc, Pensacola, FL, USA) was fixed at the waist, on the right iliac crest, using an elastic strap, programmed with one-second epochs, which were then converted to 15 seconds, using ActiLife software (version 6.8.1; ActiGraph LLC, Pensacola, FL, USA). The cut-off point of Evenson et al. ${ }^{19}$ was used to obtain the variable sedentary time (in minutes), according to a previous study that analyzed several cut-off points and found that 100 counts per minute most closely matched the ActivPAL sitting time and standing time, obtained in schoolchildren from 8 to 12 years of age during the school period ${ }^{16}$.

The inclinometer (ActivPAL - PAL Technologies Limited, Glasgow, Scotland, UK) was fixed to the anterior midline of the right thigh using adhesive tape, programmed with 15-second epochs, using ActivPAL software (version 7.2.38, PAL Technologies Limited, Glasgow, Scotland, UK). Sitting time and the sum of sitting time and standing time were considered as variables, both in minutes.

The schoolchildren were informed that the devices were used to measure their movements at school, but they did not have the information or understanding that the different body positions (sitting/reclining, standing stationary, and walking activities) and accelerations were assessed separately so that they could interfere with their usual behaviors. 
The body mass index (BMI) was calculated using weight (kilograms) and height (meters) measurements, obtained at school, using a portable scale (WISO, Crivitta Diagnóstica Ltda, São José-SC, Brazil) and stadiometer (Sanny, American Medical do Brasil Ltda, São Bernardo do Campo-SP, Brazil). The children were barefoot and, for the height measurement, in an orthostatic position, with the chin parallel to the ground and the heels together.

\section{Statistical analysis}

The Kolmogorov-Smirnov test was used to test the normality of data distribution. Comparisons between mean values of sedentary time (accelerometer) and sitting time (inclinometer), and between sedentary time (accelerometer) and the sum of sitting time and standing time (inclinometer) were performed using paired Student's ttests. Pearson's correlation coefficients were adopted to verify correlations, intraclass correlation coefficients (ICC) to verify the reliability, and Bland-Altman plots were used to visualize agreement between the same variables. The analyses were performed using SPSS (version 22.0; IBM Corp., Armonk, NY, USA) and Medcalc (MedCalc Software Ltd., Ostend, Belgium) programs. The significance level adopted was $\mathrm{p}<0.05$.

\section{Results}

The sample consisted mostly of boys, with an average age of approximately 8 years and normal body mass index for the age group ${ }^{20}$, according to the characteristics described in Table 1.

When comparing the mean values of sedentary time (ActiGraph GT3X) with sitting time (ActivPAL), the mean times were different (134.2 versus $120.3 \mathrm{~min} /$ day), the ICC was 0.608 , and the Pearson's correlation was 0.53 (Figure 1A). The Bland Altman plot showed that the ActiGraph GT3X measurement was overestimated compared to the ActivPAL (mean 12.7\%) (Figure 2A).

The measurement of sedentary time with the ActiGraph GT3X was also compared to the sum of sitting time and standing time measurements with the ActivPAL. Although the comparison presented a positive Pearson's correlation $(r=0.75)$, higher than the comparison with the

Table 1 - Descriptive statistics of the participants.

\begin{tabular}{lccc}
\hline Variables & All & Male & Female \\
\hline Sex & $60(100 \%)$ & $34(56.7 \%)$ & $26(43.3 \%)$ \\
Age (years) & $7.8(0.6)$ & $7.7(0.5)$ & $7.9(0.7)$ \\
Weight $(\mathrm{kg})$ & $26.3(5.2)$ & $25.9(3.9)$ & $26.7(6.5)$ \\
Stature $(\mathrm{m})$ & $1.25(0.06)$ & $1.26(0.04)$ & $1.25(0.07)$ \\
BMI $\left(\mathrm{kg} / \mathrm{m}^{2}\right)$ & $16.5(2.5)$ & $16.3(2.2)$ & $16.7(2.9)$ \\
\hline
\end{tabular}

Note: $\mathrm{BMI}=$ Body Mass Index. All variables are described as mean and standard deviation, except sex, which is presented using absolute and relative frequencies.

\section{A Sedentary time vs Sitting time}

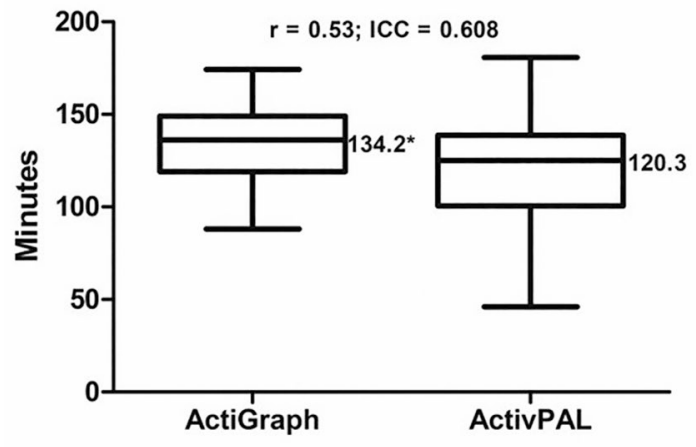

B Sedentary time vs Sitting+Standing time

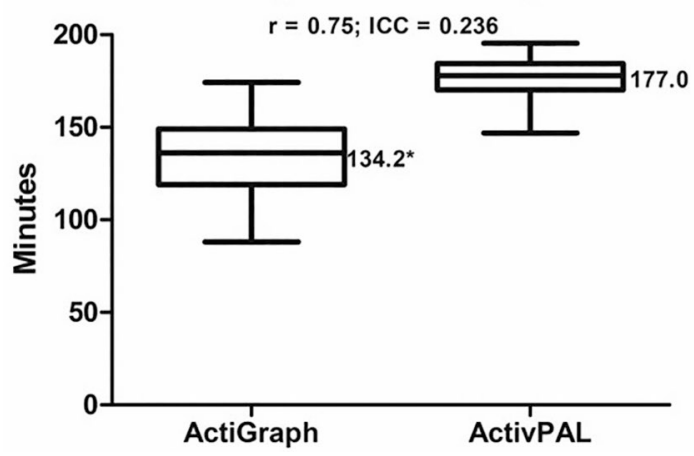

Figure 1 - Correlation and comparison of means in minutes $(95 \% \mathrm{CI})$ for ActiGraph GT3X and ActivPAL. Detailed legend: A - Sedentary time from ActiGraph GT3X versus Sitting time from ActivPAL. B - Sedentary time from ActiGraph GT3X versus Sitting and Standing time from ActivPAL. Note: $r=$ Pearson's correlation coefficients; ICC $=$ intraclass correlation coefficients; $* \mathrm{p}<0.001$ for comparison of means.

measure of sitting time alone, the ICC was smaller (0.236), and the means were also different (134.2 versus $177.0 \mathrm{~min} /$ day) (Figure 1B). The Bland Altman plot showed that the inclusion of standing time changes the estimate in $-28.4 \%$, that is, it changes from overestimation to underestimation of the ActiGraph GT3X measurement relative to the ActivPAL (mean $-42.8 \mathrm{~min} /$ day) (Figure 2B).

\section{Discussion}

We found differences in the measurements obtained through the ActiGraph GT3X compared to the ActivPAL measurements in children during the school period. The comparison of the sedentary time measurement from the ActiGraph GT3X and sitting time from the ActivPAL demonstrated a mean overestimation of approximately 14 minutes, ranging up to 60 minutes. The ActiGraph GT3X model probably overestimates sedentary time because it does not differentiate body postures, so considers standing as stationary time, which the ActivPAL can differentiate from sitting time. 
A
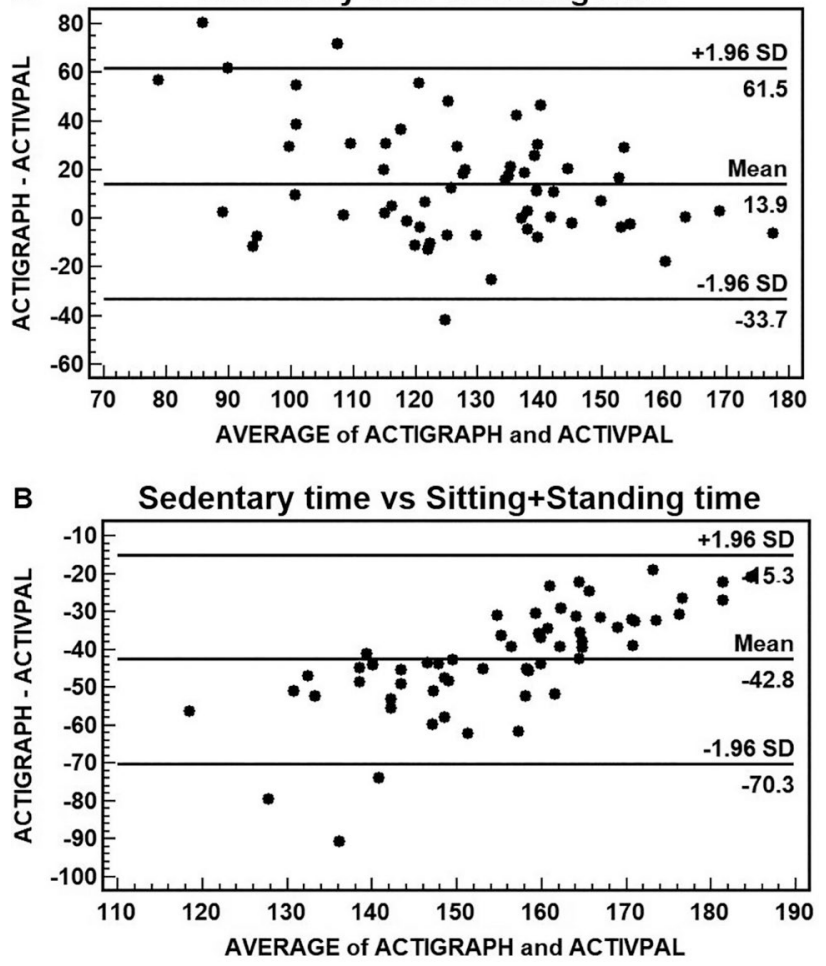

Figure 2 - Bland-Altman plots of the differences between ActiGraph GT3X and ActivPAL: A - Sedentary time from ActiGraph GT3X versus Sitting time from ActivPAL. B - Sedentary time from ActiGraph GT3X versus Sitting and Standing time from ActivPAL.

However, when adding standing time to sitting time of the ActivPAL (stationary behavior), the ActiGraph GT3X underestimated ActivPAL by approximately $43 \mathrm{~min}$, although with a stronger correlation than when considering only sitting time from the ActivPAL. This result demonstrates that the ActiGraph GT3X measurement could include some standing position activities (but not all), which cannot conceptually be classified as SB, producing an aleatory error. This may be since ActivPAL does not detect activities performed with the upper limbs and hips in a standing position, while the ActiGraph GT3X classifies some of these movements as light physical activity and not as sedentary time.

Previous studies have also found differences between the devices available for measuring SB in young people, even when using the ActiGraph inclinometer function $^{15,18}$. We found that the mean difference in the SB measurement with the ActiGraph was almost 13\% (LoA $-33 \%$ to $58 \%$ ), higher than that observed by Martin et al. ${ }^{21}$ in Scottish young people (4.3\%). We also found higher differences between devices when compared with controlled situations, given the current study was performed in a real-world context ${ }^{22-24}$. This result reinforces the potential bias associated with the use of or comparisons between evidence produced by both devices.
These differences between measures may overestimate or underestimate the real effect of interventions aimed at reducing SB in various environments, thus compromising the result and comparisons between studies ${ }^{25}$. For example, we have previously verified, in Portuguese schoolchildren from 11 to 13 years of age, that the ActiGraph GT3X does indeed seem to overestimate SB in school (16.8\%), besides not detecting changes in SB promoted by an intervention to reduce SB in school (ActivPAL as reference) ${ }^{17}$. Also, depending on the device used, SB may or may not be associated with health outcomes such adiposity, cardio-metabolic, fitness, bone/musculoskeletal, and psychosocial, gross motor, and cognitive skills, compromising the ability to correctly detect these associations ${ }^{26}$.

Ridgers et $\mathrm{al}^{16}$ also compared sedentary time measurements obtained using ActiGraph accelerometers with ActivPAL measurements in children aged 8 to 12 years in the school environment. They used several ActiGraph cutoff points and in conclusion, the authors indicated that sitting time at school can be obtained with the ActiGraph cut-off point of 100 counts per minute, and that other high cut-off points can record both standing and sitting postures by accelerometers. In our study, even using the cut-off point of 100 counts per minute, we found differences between the two devices, however, in addition to the study by Ridgers et al using another ActiGraph device model (GT1M), our students used the devices for four days, instead of two days, and for a shorter period (within a four-hour shift), which can justify the differences between the results found.

In this sense, sedentary time measured using hipworn ActiGraph GT3X accelerometers fits more into the concept of stationary behavior, which covers any motionless activity, regardless of body position and energy expenditure, while the sitting time measured using the ActivPAL would be more in line with the narrower concept of SB, especially the postural dimension ${ }^{1}$. In addition to a better understanding of the most appropriate terminology, this differentiation between what each measure represents is fundamental for understanding the results presented in previous studies that evaluated SB using these two instruments, and for choosing the best evaluation method for future studies.

One of the limitations of the present study was the fact that the children used the devices for approximately four days during the school period (7 am to $11 \mathrm{am}$ ). Different results may be found if devices are used following the standard protocol of seven consecutive days. However, the usual seven-day protocol is used for evaluation in all domains, and our study aimed to evaluate only in the school context. Another limitation was that we did not consider a direct observation measure to compare with measurements obtained through the devices. On the other hand, the present study was able to compare two devices 
through their concomitant use. Furthermore, to provide information on the measurement of SB, the study sought, using comparisons with other measures, to improve understanding of the specificity of each device to evaluate measures of SB.

\section{Conclusion}

Sedentary time measured with the ActiGraph GT3X, despite positively correlating with sitting time and the sum of sitting time and standing time from the ActivPAL, should be used with caution to evaluate sedentary behavior in a school setting and can be interpreted only as activities without movement (stationary behavior). Future studies should investigate which activities in a standing position are considered sedentary by accelerometers such as the ActiGraph GT3X device. The correct identification of sedentary time at school and its differentiation from other types of behaviors should be taken into account for future studies in the school environment, looking for associations between sedentary behaviors and academic outcomes, as well as in studies that seek to assess the effects of interventions to reduce sitting time at school.

\section{Acknowledgments}

André Werneck is supported by the São Paulo Research Foundation (FAPESP) with a Ph.D. scholarship (FAPESP process: 2019/24124-7). We gratefully acknowledge the contribution of all participating, school principal, coordinator, and teachers, as well as the Municipal Secretariat of Education of Aracaju. We thank the International Society of Behavioral Nutrition and Physical Activity for supporting the wider project through the Pioneer Scholarship Grant. We also thank the Laboratory of the Study and Research Group in Metabolism, Nutrition, and Exercise (GEPEMENE) from Londrina State University for lending the devices (accelerometers and inclinometers) to this study. And we thank Jaqueline Reis and Lucas Souza for their contributions to the discussion of the results of the manuscript. The views expressed in this publication are those of the authors and not necessarily those of the acknowledged institutions.

\section{References}

1. Tremblay MS, Aubert S, Barnes JD, Saunders TJ, Carson V, Latimer-Cheung AE, et al. Sedentary Behavior Research Network (SBRN) - Terminology Consensus Project process and outcome. Int J Behav Nutr Phys Act. 2017;14(1):1-17. doi

2. Chudasama YV, Khunti KK, Zaccardi F, Rowlands AV, Yates T, Gillies CL, et al. Physical activity, multimorbidity, and life expectancy: a UK Biobank longitudinal study. BMC Med. 2019;17(1):108. doi
3. Ekelund U, Steene-Johannessen J, Brown WJ, Fagerland MW, Owen N, Powell KE, et al. Does physical activity attenuate, or even eliminate, the detrimental association of sitting time with mortality? A harmonised meta-analysis of data from more than 1 million men and women. Lancet Lond Engl. 2016;388(10051):1302-10. doi

4. Katzmarzyk PT, Powell KE, Jakicic JM, Troiano RP, Piercy $\mathrm{K}$, Tennant B, et al. Sedentary behavior and health: update from the 2018 Physical Activity Guidelines Advisory Committee. Med Sci Sports Exerc. 2019;51(6):1227-41. doi

5. Carson V, Hunter S, Kuzik N, Gray CE, Poitras VJ, Chaput $\mathrm{J}-\mathrm{P}$, et al. Systematic review of sedentary behaviour and health indicators in school-aged children and youth: an update. Appl Physiol Nutr Metab Physiol Appl Nutr Metab. 2016;41(6):S240-65. doi

6. Wu XY, Han LH, Zhang JH, Luo S, Hu JW, Sun K. The influence of physical activity, sedentary behavior on healthrelated quality of life among the general population of children and adolescents: a systematic review. PloS One. 2017;12(11):e0187668. doi

7. Rodriguez-Ayllon M, Cadenas-Sánchez C, Estévez-López F, Muñoz NE, Mora-Gonzalez J, Migueles JH, et al. Role of physical activity and sedentary behavior in the mental health of preschoolers, children and adolescents: a systematic review and meta-analysis. Sports Med Auckl NZ. 2019;49(9):1383-410. doi

8. Hardy LL, Hills AP, Timperio A, Cliff D, Lubans D, Morgan PJ, et al. A hitchhiker's guide to assessing sedentary behaviour among young people: deciding what method to use. J Sci Med Sport. 2013;16(1):28-35. doi

9. Sanders JP, Loveday A, Pearson N, Edwardson C, Yates T, Biddle SJH, et al. Devices for self-monitoring sedentary time or physical activity: a scoping review. J Med Internet Res. 2016;18(5):e90. doi

10. Atkin AJ, Gorely T, Clemes SA, Yates T, Edwardson C, Brage S, et al. Methods of Measurement in epidemiology: sedentary behaviour. Int J Epidemiol. 2012;41(5):1460-71. doi

11. Grant PM, Ryan CG, Tigbe WW, Granat MH. The validation of a novel activity monitor in the measurement of posture and motion during everyday activities. Br J Sports Med. 2006;40(12):992-7. doi

12. Migueles JH, Cadenas-Sanchez C, Ekelund U, Delisle Nyström C, Mora-Gonzalez J, Löf M, et al. Accelerometer data collection and processing criteria to assess physical activity and other outcomes: a systematic review and practical considerations. Sports Med Auckl NZ. 2017;47 (9):1821-45. doi

13. Gibbs BB, Hergenroeder AL, Katzmarzyk PT, Lee I-M, Jakicic JM. Definition, measurement, and health risks associated with sedentary behavior. Med Sci Sports Exerc. 2015;47(6):1295-300. doi

14. Trost SG, Pate RR, Sallis JF, Freedson PS, Taylor WC, Dowda $\mathrm{M}$, et al. Age and gender differences in objectively measured physical activity in youth. Med Sci Sports Exerc. 2002;34(2):350-5. doi

15. De Decker E, De Craemer M, Santos-Lozano A, Van Cauwenberghe E, De Bourdeaudhuij I, Cardon G. Validity of the ActivPAL ${ }^{\mathrm{TM}}$ and the ActiGraph monitors in preschoolers. Med Sci Sports Exerc. 2013;45(10):2002-11. doi 
16. Ridgers ND, Salmon J, Ridley K, O’Connell E, Arundell L, Timperio A. Agreement between activPAL and ActiGraph for assessing children's sedentary time. Int J Behav Nutr Phys Act. 2012;9(1):1-8. doi

17. Silva DR, Minderico CS, Júdice PB, Werneck AO, Ohara D, Cyrino ES, et al. Agreement between GT3X accelerometer and ActivPAL inclinometer for estimating and detecting changes in different contexts of sedentary time among adolescents. J Phys Act Health. 2019;16(9):780-4. doi

18. Ridley K, Ridgers ND, Salmon J. Criterion validity of the activPAL ${ }^{\mathrm{TM}}$ and ActiGraph for assessing children's sitting and standing time in a school classroom setting. Int J Behav Nutr Phys Act. 2016;13(1):1-5. doi

19. Evenson KR, Catellier DJ, Gill K, Ondrak KS, McMurray RG. Calibration of two objective measures of physical activity for children. J Sports Sci. 2008;26(14):1557-65. doi

20. World Health Organization. BMI-for-age (5-19 years). Available from: http://www.who.int/growthref/who2007_b mi_for_age/en/ [accessed 7th April 2020].

21. Martin A, McNeill M, Penpraze V, Dall P, Granat M, Paton JY, et al. Objective measurement of habitual sedentary behavior in pre-school children: comparison of activPAL With Actigraph monitors. Pediatr Exerc Sci. 2011;23 (4):468-76. doi

22. Dowd KP, Harrington DM, Donnelly AE. Criterion and concurrent validity of the activPAL ${ }^{\mathrm{TM}}$ professional physical activity monitor in adolescent females. PloS One. 2012;7 (10):e47633. doi

23. Alberto F-P, Nathanael M, Mathew B, Ainsworth BE. Wearable monitors criterion validity for energy expenditure in sedentary and light activities. J Sport Health Sci. 2017;6 (1):103-10. doi
24. Stålesen J, Vik FN, Hansen BH, Berntsen S. Comparison of three activity monitors for estimating sedentary time among children. BMC Sports Sci Med Rehabil. 2016;8(1):1-6. doi

25. Kozey-Keadle S, Libertine A, Lyden K, Staudenmayer J, Freedson PS. Validation of wearable monitors for assessing sedentary behavior. Med Sci Sports Exerc. 2011;43 (8):1561-7. doi

26. Cliff DP, Hesketh KD, Vella SA, Hinkley T, Tsiros MD, Ridgers ND, et al. Objectively measured sedentary behaviour and health and development in children and adolescents: systematic review and meta-analysis. Obes Rev Off J Int Assoc Study Obes. 2016;17(4):330-44. doi

\section{Corresponding author}

Luciana Leite Silva Barboza. Universidade Federal de Sergipe, Programa de Pós-Graduação em Educação Física, Av. Marechal Rondon, Rosa Elze, 49100-000, São

Cristóvão, SE, Brazil.

E-mail: leite.lu@gmail.com.

Manuscript received on July 17, 2021

Manuscript accepted on December 9, 2021

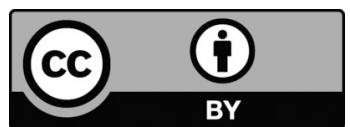

Motriz. The Journal of Physical Education. UNESP. Rio Claro, SP, Brazil - eISSN: 1980-6574 - under a license Creative Commons - Version 4.0 